
allemande

47-2 | 2015

Retour sur le modèle Rhénan : humanisme, capitalisme et métropolisation | Patrimonialisation du passé « allemand » en Europe centrale après 1990

\title{
Préserver et promouvoir l'humain dans la vie socio-économique
}

Un témoignage du Forum humaniste rhénan

Sabine Urban

\section{(2) OpenEdition}

Édition électronique

URL : https://journals.openedition.org/allemagne/296

DOI : 10.4000/allemagne.296

ISSN : 2605-7913

Éditeur

Société d'études allemandes

\section{Édition imprimée}

Date de publication : 16 décembre 2015

Pagination : 349-359

ISSN : 0035-0974

\section{Référence électronique}

Sabine Urban, « Préserver et promouvoir l'humain dans la vie socio-économique », Revue d'Allemagne et des pays de langue allemande [En ligne], 47-2 | 2015, mis en ligne le 13 décembre 2017, consulté le 22 mai 2021. URL : http://journals.openedition.org/allemagne/296 ; DOI : https://doi.org/10.4000/ allemagne.296 


\section{Préserver et promouvoir l'humain dans la vie socio-économique \\ Un témoignage du Forum humaniste rhénan ${ }^{1}$}

\section{- Sabine Urban*}

Le fondement de cette recherche est double: il repose sur un constat et sur une volonté.

Le constat, qui s'exprime avec une acceptation croissante dans le monde, est que l'évolution de l'économie globalisée, que l'on peut observer depuis la fin des années 1980, allant dans le sens d'une financiarisation en progression rapide de la vie sociale, n'est pas « soutenable » dans la durée car elle porte en elle les germes d'affaiblissement de valeurs humaines au profit de la toute-puissance de l'argent, d'inégalités sociales excessives, de situations d'endettement public ingérables et de massacres environnementaux suicidaires.

La volonté de réagir à cette évolution, par la réflexion et des propositions d'action, est la marque de fabrique du Forum humaniste rhénan, créé à l'initiative de Pierre Karli, neurobiologiste à l'Université de Strasbourg et membre de l'Académie des Sciences, qui s'est entouré à cette occasion d'un certain nombre de personnalités scientifiques, politiques, de la vie sociale et du monde des affaires pour déterminer une série de thématiques appropriées. La première (Préserver et promouvoir l’humain dans la vie

* Professeur émérite de l'Université de Strasbourg. Présidente du Forum humaniste rhénan/Humanismus am Oberrhein (www.fhr.fho.unistra.fr).

1 Cet article a été conçu à la suite du Séminaire franco-allemand qui s'est tenu à Strasbourg le 12 octobre 2013 et qui a réuni autour de deux Tables rondes les personnalités françaises et allemandes suivantes: Brigitte Bannerot (médecin inspecteur régional du travail), Astrid Boos (présidente du Conseil d'administration et de surveillance de la Caisse d'Épargne d'Alsace), Maximilian Erlmeier (président de la Freiburger Denkfabrik), Gert Fieguth (professeur à la Hochschule de Kehl), Patrick Guillot (directeur général des Hôpitaux universitaires de Strasbourg), André Le Bihan (dirigeant de Siemens France), Karsten Lehmkühler (professeur à I'Université de Strasbourg), Geneviève Lepelletier (directrice des Ressources humaines du Groupe Socomec), Hans Nussbaum (chef d'entreprise, Nussbaum Gruppe), Norbert Rauch (chef d'entreprise, Rauch Landesmachinenfabrik), Fabrice Urban (président directeur général de Quiri et Cie). 
socio-économique) a été débattue durant une journée de séminaire à la Maison de la Région à Strasbourg, le samedi 12 octobre 2013. Une des particularités de ce séminaire était que le débat était animé, à parité, par des modérateurs et des acteurs de terrain de part et d'autre du Rhin (avec traduction simultanée).

Le message présenté dans les pages qui suivent n'est pas un compte-rendu analytique explicite des différentes interventions, mais une note de synthèse des pensées émises, volontairement brève pour être susceptible de stimuler ultérieurement des réactions et des idées fécondes dans différentes sphères de la vie sociale.

Ce message a été forgé au sein du Forum humaniste rhénan dont la dénomination évoque à la fois une histoire et un espace. L'histoire remonte à la "Renaissance » qui a engagé l'Europe aux $\mathrm{XV}^{\mathrm{e}}$ et $\mathrm{XVI}^{\mathrm{e}}$ siècles dans une phase de rayonnement culturel, scientifique, spirituel et économique brillante. L'espace rhénan y contribue d'une manière particulière sous la double influence de la Renaissance (en tant que ferment de la modernité) et de la Réforme protestante (développant l'analyse critique et le sens de la responsabilité). Ces particularités ont notamment engendré, chemin faisant, des réalités bien vivantes telles que l'« économie sociale de marché » (développée par l'école de pensée ordo-libérale de Fribourg-en-Brisgau), ou le courant «Neue Wege des Humanismus / Nouveaux chemins de l'humanisme " né à Bâle en 1996, ou plus récemment encore l'initiative de Maximilian Erlmeier (Fribourg-en-Br.) pour une "Humane Marktwirtschaft / Économie de marché humaine ».

Le présent porte la marque du passé, mais ce qui importe c'est le futur; celui-ci se construit au présent, sur la base d'idées, d'expériences, de projets. C'est dans cet esprit que les organisateurs du séminaire Préserver et promouvoir l'humain dans la vie socioéconomique ont demandé aux participants, tous acteurs de terrain dans des organisations diverses, de livrer leur témoignage de pratiques effectives, leur perception de la réalité socio-économique, leur vision de l'évolution vers un futur « soutenable » dans le long terme des générations futures, et « souhaitable » au regard du bien-être et de la dignité des hommes, de tous les hommes.

En d'autres termes, il s'agit de penser la finalité de l'économie. Dans le courant humaniste, c'est l'élément humain, c'est-à-dire l'homme, qui mérite d'être placé au cœur des préoccupations du système socio-économique, par opposition à un autre courant de pensée en vogue qui préfère y voir l'argent, le rendement financier (une donnée relative) et le gain monétaire (une donnée absolue). Le choix de l'une ou l'autre priorité n'est pas neutre; il modifie en fait le fonctionnement du système dans son ensemble. N'oublions pas, cependant, que pour créer les richesses (les biens et les services) nécessaires à la survie et à l'épanouissement de l'homme, le système productif a besoin à la fois de la ressource humaine, dans toute sa diversité de talents, de compétences, de capacités créatrices, et d'argent c'est-à-dire de ressources financières pour investir, innover, rémunérer le travail et contribuer aux recettes du secteur public. C’est dire que le choix de la priorité (valeur humaine versus valeur monétaire) doit être mesuré subtilement. L'entreprise se trouve placée au cœur de ce dilemme: les grandes entreprises multinationales et les PME (petites et moyennes entreprises) ne font pas leurs choix avec les mêmes objectifs et les mêmes contraintes; les grandes, cotées en Bourse, sont directement dépendantes des marchés financiers a-nationaux (qui jugent 
prioritairement en termes d'optimisation des résultats financiers à court terme), les secondes, souvent liées à des patrimoines familiaux, sont plus proches des gens (et sont dès lors plus sensibles au devenir du « capital » humain). Par ailleurs, les choix varient selon le contexte culturel, social et politique des entreprises. Cette évidence s'est dégagée très nettement des discussions de la journée; un clivage existe bel et bien de part et d'autre du Rhin. Le substrat humaniste commun a été infléchi par le centralisme parisien, d'un côté, par l'acceptation d'une diversité autonome, de l'autre.

Les débats du séminaire du 12 octobre 2013 ont été centrés plus précisément sur deux thèmes: le premier concerne la quête de sens et le besoin de reconnaissance, le second les relations et la communication verticales et horizontales au sein du monde du travail. Au-delà d'une apparente technicité, ces deux thèmes ont été analysés avec une grande ouverture d'esprit pour prendre en considération des problèmes managériaux et éthiques plus vastes.

\section{L'homme a besoin de trouver un sens à son existence: c'est une quête person- nelle, mais le contexte socio-économique dans lequel il évolue peut faciliter (ou non) cette recherche}

L'homme est à la fois un individu biologique qui a son existence propre, ses capacités et ses besoins particuliers, son équilibre intérieur, et un acteur social qui a des besoins d'ordre relationnel et affectif, et qui ne peut pas assurer sa survie sans l'appui des autres. Autonomie et dépendance sont donc en étroite interaction durant toute la vie, mais avec des degrés d'intensité variables selon les âges. L'homme trouve sa dignité et sa force dans la pensée (Blaise Pascal); c'est la pensée (plutôt que le travail) qui donne un sens à la vie. L'homme, contrairement à l'animal, est en mesure de penser son avenir, d'inventer, d'innover, d'agir. Mais l'homme, vivant en société, est aussi un être fragile dans la mesure où il risque d'être écrasé par les pensées et les forces des autres. Des asymétries de pouvoir entre la sphère individuelle et la sphère collective existent, et sont en mesure de créer des tensions dommageables à la fois pour l'individu et le groupe. Il appartient aux acteurs du système socio-économique de les limiter. Le thème du "comment » sera évoqué tout au long des discussions, avec des variations diverses, telles que le respect des besoins des uns et des autres, la solidarité, l'écoute, le regard, le dialogue, l'ouverture à la nouveauté, l'accès au savoir sous toutes ses formes, le droit à l'erreur, la confiance, la reconnaissance, l'acceptation de la responsabilité, la liberté d'esprit critique, la souplesse d'adaptation. Ces "variations », essentiellement comportementales, déclinées par tous les participants, sont en mesure d'être pratiquées dans toutes les organisations: entreprises, hôpitaux, administrations publiques, institutions éducatives, associations, c'est-à-dire dans toutes les parties d'un système socio-économique, sans que celui-ci ne soit remis en cause de fond en comble.

L'efficacité d'un système socio-économique est la résultante d'initiatives individuelles ou collectives et des conditions de mise en valeur de ces initiatives dans le cadre d'entreprises, ou d'autres organisations. Le travail prend dans la vie des individus en âge d'être actifs beaucoup d'importance, d'abord par le temps passé, mais aussi comme forme de valorisation de leur énergie (se sentir utile), ou comme source de sécurité (par le biais d'un emploi si possible stable) ou encore comme source de lien social, voire comme 
source de plaisir. L'employeur, lui, voit dans le travail de ses collaborateurs une ressource, un facteur de production valorisée sur les marchés, mais cette ressource n'est pas seulement opérationnelle dans le court terme; elle porte en elle le devenir de l'entreprise par la capacité créatrice propre à chaque être humain. Les interactions sociales sont facteurs de progrès cognitifs. Celles-ci se développent, pour partie, au sein de l'entreprise et sont dès lors source de compétitivité pour celle-ci et facteur de valorisation de son précieux " capital humain ». Pour partie, ces interactions se réalisent aussi dans l'environnement socio-économique de l'entreprise; c'est ainsi que se créent des réseaux de toutes sortes (interpersonnels, inter-organisationnels, technologiques, scientifiques, interindustriels, voire socio-politiques) de la plus haute importance pour le développement d'une entreprise, petite ou grande. Le « travailleur", quel que soit son rang ou sa fonction, est donc à considérer à la fois comme un coût de production et un investissement d'avenir. La réalité de cette dualité coût/investissement peut être perçue de manière diverse par la hiérarchie ou la gouvernance de l'entreprise. Selon les cas, c'est le climat social dans l'entreprise qui différera. La non-perception du facteur « investissement d'avenir » peut hélas entraîner des dérives de gestion se traduisant dans les cas extrêmes (plus fréquents dans les grands groupes que dans les PME) par le désespoir, le mal-être, des tendances dépressives des travailleurs ou des phénomènes de "burn out ", possiblement dramatiques. Le travail peut alors s'apparenter à un «non-sens".

En tout état de cause, le travail n'est pas la seule source capable de nourrir la quête de sens d'un individu. La vie existe en dehors de la sphère du travail et de l'entreprise (ou autre organisation) qui l'offre. L'idée que l'entreprise doive répondre directement à ce besoin de sens recherché par les individus a été largement réfutée, pour au moins deux séries de raisons. La première est que l'entreprise n'est pas une « secte » destinée à endoctriner ses collaborateurs; elle a fondamentalement une autre mission, celle de créer des richesses répondant aux besoins des individus (clients, consommateurs, usagers, utilisateurs industriels, collectivités); il convient de reconnaître aux collaborateurs la liberté de penser et de construire le sens qu'ils entendent donner à leur vie. La seconde est que la quête de sens, exprimée en général plus ou moins confusément, est susceptible de subir des manipulations managériales (de groupes ou de nature politique comme on a pu l'observer dans des régimes à tendance totalitaire, mais pas seulement) fort contraignantes, voire dommageables. La quête de sens concerne d'abord la vie intime de chacun. Il faut la respecter en tant que telle. Il appartient par contre à l'entreprise la responsabilité de ne pas malmener la liberté de choix personnel. Il faut que l'entreprise, créatrice de richesse, rende possible l'émergence de sens pour l'individu. Le lien entre travail et création de valeur peut et doit être souligné par le management. C'est ainsi que l'une des entreprises participantes, spécialisée dans le machinisme agricole, a développé une culture d'entreprise centrée sur la satisfaction des besoins nutritionnels de l'humanité (la partie dominante de la production de machines étant exportée dans le vaste monde) et la contribution au développement durable. A contrario, il est bien connu qu'il existe aussi des entreprises broyeuses de sens et pourtant très profitables (du moins à court terme). Dans une vision plus durable d'un capitalisme à visage humain, les convictions « humanistes » doivent être portées avec une très grande cohérence entre l'actionnariat, les entreprises et l'ensemble des équipes et personnes qui constituent l'entreprise: c'est l'actionnariat qui donne le ton de la «financiarisation » ou non du pilotage de l'entreprise; ce sont les dirigeants qui 
sont susceptibles de forger une vision claire et crédible de la manière de vivre et de travailler ensemble qu'ils prônent pour l'entreprise; c'est l'ensemble des équipes et des personnes constitutives de l'entreprise qui a la responsabilité importante de proposition, d'action et d'initiative. Le dialogue social a toute sa place dans pareil dispositif.

Dans cette perspective, il convient de réfléchir aux éléments qui facilitent la vie au travail, c'est-à-dire la capacité (Fähigkeit) pour l'individu d'exercer un travail, profitablement pour lui et pour l'entreprise (ou organisation sans but lucratif) et sa disponibilité ou bonne volonté (Bereitschaft) pour exercer un travail. Ces éléments sont conditionnés pour chacun, plus particulièrement, par son bon état de santé, par sa joie de vivre et par sa motivation. Pour le premier élément, l'entreprise y est pour quelque chose et sa responsabilité est encadrée par la médecine du travail. Pour le deuxième élément, l'entreprise n'est responsable que d'une faible part. La joie de vivre manifestée par un individu relève d'éléments caractériels propres liés à son milieu affectif, à son équilibre intérieur, à son éducation, au cadre social et culturel dans lequel il évolue. Il n'empêche que cette joie de vivre se manifeste aussi lorsque l'individu a confiance en lui; cette confiance, il la doit souvent à la qualité de sa formation, à même de lui donner de l'assurance dans la vie et de le persuader de son aptitude à pouvoir profiter des opportunités offertes chemin faisant. En l'occurrence, le système allemand de formation duale (alternant présence en entreprise et à l'école) et la reconnaissance communément accordée à l'apprentissage (un apprentissage chez Daimler Benz ou Siemens ou BASF, etc. vaut une entrée garantie dans la vie active, voire une possibilité de capillarité sociale jusqu'au sommet de la hiérarchie) sont globalement plus performants que le système de formation professionnelle français, très préoccupé par la formation des élites mais laissant sur le bord du chemin vers le marché du travail un nombre intolérable de jeunes en âge de travailler, réduits au chômage ou à la précarité. Mais il faut bien être conscient que les systèmes de formation sont enracinés dans des traditions et des comportements sociaux, liés à des jugements de valeur spécifiques qui portent la marque de l'histoire des territoires; ils ne s'imposent pas, ou ne se copient pas du jour au lendemain. L'apprentissage en France manque de souffle, de cohérence politique et plus généralement de reconnaissance, et ce depuis un certain temps. Pour le troisième élément cité, la motivation, l'entreprise joue un grand rôle. La motivation dépend notamment de la qualité du travail offert, de la responsabilité accordée, de la reconnaissance exprimée, du climat régnant dans l'entreprise, des relations avec les autres au sein de l'organisation, de la participation à la vie du groupe. La direction de l'entreprise joue en la matière un rôle majeur. Elle donne le « la » de cette musique sociale.

Il existe cependant des éléments qui dépassent le cadre de compétence et de responsabilité propre de telle ou telle entreprise (ou organisation).

\section{Des bouleversements majeurs sont en cours}

Un changement de paradigme se dessine avec l'intrusion dans toutes les sphères de la vie sociale des technologies numériques et d'un nouveau pouvoir, celui de Big Data, un « être » imprécis, à part, une partie prenante omnisciente et invasive qui crée l'asymétrie de pouvoir majeure des temps modernes. L'intrusion de la machine qui a caractérisé la "révolution industrielle " (magnifiquement illustrée dans le film de Charlie Chaplin, Les temps modernes) a démultiplié la productivité du facteur travail, associé 
au facteur capital qu'est la machine, et mis à la disposition de l'humanité le bien-être matériel. Mais le travail en entreprise était rythmé par des temps séparés: celui du travail d'une part, celui du repos et du loisir d'autre part; le premier relevant de la sphère de l'organisation collective, le second du libre-arbitre individuel. Aujourd'hui, cette séparation du temps est balayée: Big Data vient perturber la vie quotidienne (de travail ou de loisir) de tout un chacun en s'infiltrant nuit et jour dans la vie privée. Où se déroulera à l'avenir le dialogue social (devant l'écran de l'ordinateur, de la tablette, à l'écoute d'un smartphone)? Comment vont se résoudre les conflits interpersonnels ou sociaux? Comment va-t-on négocier un consensus ou un compromis? Quand trouvera-t-on le temps de dormir? de réfléchir? de vivre sa vie personnelle avec le sens qu'on veut lui trouver? Comment échapper au stress dans un contexte technologique vivace et actif 24 heures sur 24 , et qui dès lors impose sa forme de tyrannie à la planète entière, sans retenue ou ménagement et donnant mauvaise conscience à ceux qui ne suivent pas le rythme? Que peut-on ou doit-on encore réguler? Quelle forme d'expression démocratique est-elle envisageable? La valeur sûre, jusqu'à présent, du dialogue interpersonnel, du regard, du partage, est-elle encore de mise? Le changement piétine à la porte.

\section{Une aspiration constante: celle de la reconnaissance}

L'homme reste un individu qui a besoin de vivre sa vie, avec une certaine autonomie et un minimum de liberté. Il a aussi besoin d'être rassuré, sur lui-même et sur sa relation à l'autre.

$\mathrm{La}$ « reconnaissance » prend dès lors de multiples formes. C'est d'abord le droit d'exister. L'individu, tout individu, a besoin de se sentir reconnu comme un élément de la vie sociale. La langue allemande dispose d'une belle expression pour souligner cette relation, "auf Augenhöhe » c'est-à-dire d'égal à égal, à hauteur des yeux, au même niveau d'humanité, sans qu'intervienne une distance hiérarchique potentiellement blessante ou méprisante en termes de dignité humaine. La parité du dialogue a son importance. La reconnaissance du travail est identifiée comme un facteur modérateur du stress. En matière de reconnaissance, le besoin fondamental de l'individu au travail (donc dans la vie socio-économique) est de se reconnaître soi-même dans le miroir de son travail. Mettre «quelque chose de soi » dans son travail est une aspiration commune à toutes les personnes en activité, dans une entreprise productive ou dans un autre cadre. Dans un hôpital, par exemple, il est important de développer la reconnaissance du rôle transversal des différents groupes professionnels: médecins, infirmiers, personnel paramédical, directeurs, tous confrontés à la fragilité de la condition humaine, de la naissance à la mort, en passant par les souffrances, la douleur, la solitude, tous appelés à œuvrer ensemble. La reconnaissance va contribuer à apporter un progrès pour la qualité des soins et, par la suite, la réduction de l'angoisse des malades. Dans une grande entreprise multinationale, la reconnaissance des uns et des autres passera par l'explicitation du rôle de chacun dans un projet industriel. L'implication de tous est nécessaire car il existe des problèmes à résoudre à tous les niveaux, du sommet à la base. Bien des solutions de bon sens, perspicaces, sont imaginées au niveau du terrain opérationnel et non pas dans un bureau d'étude. La reconnaissance implique aussi la prise de conscience que pour chaque collaborateur, il faut éviter le gaspillage de son 
temps (en réunions inutiles, mal préparées ou redondantes) et de son énergie, un bien précieux pour la dynamique de l'ensemble. La reconnaissance individuelle passe par l'implication du chef, sa proximité et sa disponibilité. Le chef, dans son domaine de responsabilité, doit connaître les acteurs concernés, savoir ce qu'ils apportent et leur montrer que la direction est sensible à leur engagement. La reconnaissance est une clé de la motivation au travail; elle est complémentaire à la valorisation du travail par le salaire et les primes. Globalement, au niveau de l'entreprise, une pratique de reconnaissance stimule l'efficacité économique; l'inverse est vrai. Dans une organisation productive, tous les collaborateurs participent finalement à la réussite financière de l'entreprise, ce qui logiquement donne lieu à un comportement respectueux et coopératif entre tous les niveaux hiérarchiques. Chaque individu est nécessaire à l'achèvement de l'objectif commun (la stratégie). L'engagement de chacun suscite sa reconnaissance qui conduit à son tour à un haut niveau de satisfaction au travail qui se solde finalement par un faible taux de rotation (turnover) du personnel. Ainsi, le savoir acquis reste valorisé dans l'entreprise et le « capital humain » ne se trouve pas dispersé. La réussite de l'entreprise, dans le cas d'une politique de reconnaissance bien conduite, entretient finalement une identité de fierté partagée entre tous les membres, qui sera à son tour garante de sa dynamique. À l'opposé, dans une organisation sans politique de reconnaissance, on aboutira à des pratiques de " gestion des inerties » sans avenir. D'un point de vue philosophique la « lutte pour la reconnaissance » est un concept fondamental de la pensée sociale. D’un point de vue pratique, la reconnaissance est liée à la communication. Mais la politique et la pratique de celle-ci ont une envergure bien plus étendue. Qui dépasse largement le cadre de la reconnaissance.

\section{La communication, l'alpha et l'oméga des temps modernes}

La communication représente un secteur d'activité créateur d'emplois, en forte croissance, à un rythme rapide. Personne (individu ou organisation) ne semble pouvoir y échapper, avec le risque de susciter ou de subir une overdose. L'envahissement de la «com» suggère qu'elle est absolument essentielle à la vie socio-économique. Cela se vérifiera. Mais le phénomène suscite aussi des interpellations sur son mode d'emploi, sur de possibles abus.

La communication est un outil de gestion puissant qui a le pouvoir positif de stimuler l'énergie humaine, de créer et de modifier des images, de faire émerger de la nouveauté.

La communication a un sens multiple selon la forme qui lui est conférée: verticale, horizontale ou transversale, externe ou interne. La première, verticale, descendante (" top down») ou montante ("bottom up») est de nature plutôt hiérarchique: on transmet des informations, des ordres, des recommandations, des réclamations. La deuxième, horizontale, s'exprimant au niveau d'un groupe, d'un service, d'un projet se prête davantage à la discussion, au partage d'une pensée ou d'un ressenti, à des suggestions. La troisième, transversale ou diagonale, est plutôt d'essence coopérative et dynamique; on confronte des données ou des expériences diverses, on les évalue, on envisage de les transposer utilement. Ces différentes formes ne s'excluent pas l'une l'autre; elles ont toutes leur raison d'être. Encore faut-il se poser la question de savoir si elles sont toutes vraiment nécessaires car il est bien évident que la communication 
a un prix, en temps et en argent. La communication interne concerne essentiellement, pour ne pas dire exclusivement, une institution, organisation ou groupe donné, tandis que la communication externe vise à toucher les parties prenantes extérieures à l'organisation (les informer, les séduire, les convaincre, les engager, voire les rassurer).

Bien des questions se posent à l'occasion du maniement des outils de communication, délicats par nature en raison de l'impact direct ou indirect que leur utilisation peut avoir. Elles ont été éclairées par des observations de terrain.

La communication peut être considérée à la fois comme un "problème » et comme un « remède ». Il apparaît bien souvent que les problèmes de communication ne sont pas la cause mais plutôt le résultat de dysfonctionnements dans l'entreprise. Il faut donc bien comprendre pourquoi il faut communiquer. Recherche d'un consensus, d'un compromis? C'est souligner que la communication suppose un travail collectif, antérieur à un accord, à un changement.

La communication interne est facilitée lorsque règne un climat de confiance dans l'entreprise. La confiance est soutenue par la «transparence » des relations interpersonnelles et des règles du jeu en vigueur car elle limite la tentation de suspicion. La transparence permet de réduire les conflits et donc de réduire le besoin de communication réparatrice de "problèmes".

La communication externe a toute son importance dans un monde concurrentiel où il faut continuellement oxygéner la compétitivité de l'entreprise. L’opération de communication revient à expliciter aux parties prenantes les avantages compétitifs de l'entreprise ainsi qu'à rendre celle-ci plus attrayante. On parle aujourd'hui de «guerre des talents ", c'est-à-dire que chaque entreprise cherche à attirer les meilleures têtes, celles qui ont de bonnes idées pour construire l'avenir. La vision qu'ont les jeunes de leur avenir est importante. La mise en forme d'une politique attractive dépend, au moins pour partie, d'une bonne information sur la perception de l'entreprise par les autres et de leurs attentes. La consultation des réseaux sociaux (Facebook, Twitter) offre aux responsables de la communication de l'entreprise la possibilité de comprendre quels sont les facteurs de valorisation appréciés ou critiqués et, par la suite, de mettre en place une communication efficace.

La communication n'a pas à se laisser aller à un prêchi-prêcha moralisateur; l'observation honnête de la réalité concrète est bien plus convaincante. L'observation des faits réels est source de connaissance, et lorsqu'elle se combine dans une organisation avec un partage de l'information, elle peut stimuler le transfert de bonnes pratiques observées. C'est ainsi une source de créativité organisationnelle. Ceci vaut pour une petite entreprise privée comme pour un grand hôpital public.

L'un des dirigeants participant à la table ronde a affirmé que « la communication est le sang de l'entreprise ", c'est-à-dire un facteur vital du développement de l'entreprise. C'est dès lors au chef d'entreprise de s'en occuper, pas seulement en donnant des instructions mais en suivant ce qui se passe sur le terrain, aux différents niveaux concernés; non pas nécessairement pour convaincre, mais pour comprendre. Un regard, une écoute, un échange de sourire, une perception d'étonnement, de désapprobation, une rencontre dans un couloir ou près d'un poste de travail peuvent être plus éclairants qu'un long rapport de situation. Cependant, il ne faut pas sous-estimer la complexité de la communication horizontale entre salariés; elle n'est pas simple. Pour la rendre 
plus sereine, ce même chef d'entreprise (une entreprise allemande de taille moyenne, très performante) a créé un poste de «Betriebssorger » totalement autonome par rapport à la hiérarchie, chargé de prendre soin du bon climat social dans l'entreprise et de travailler avec tous les salariés à la culture de l'entreprise. La bonne communication, en interne, s'en trouve facilitée.

La communication interne de l'entreprise doit sans doute être balisée, avec des procédures institutionnelles claires, mais il faut aussi laisser une place à l'initiative spontanée. C’est ainsi que dans l'entreprise évoquée dans les lignes précédentes, le chef d'entreprise organise un "open-space " (un espace de rencontre ouvert) tous les samedis matin: chaque collaborateur est libre, s'il en a envie, de venir discuter, échanger des propos, faire des suggestions. C'est un lieu et un moment d'inclusion du salarié dans son organisation de travail. La pratique de l'open-space, un espace ouvert mais aussi de configuration mobilière adaptable (sans aménagements architecturaux vissés au sol, laissant apparaître des degrés hiérarchiques ou de préséance figés comme dans un amphithéâtre), est également recherchée dans le secteur public, pour mettre en œuvre des rencontres avec des participants désireux d'avoir des relations de discussion directes " miteinander» (tous ensemble) plutôt que "nebeneinander» (l'un à côté de l'autre). Ce qui est vrai pour l'intégration des salariés dans un groupe, l'est également pour les citoyens (qui ont envie de se sentir égaux et de pouvoir participer d'égal à égal au dialogue civique). L'adhésion à une cause ou l'efficacité d'une procédure opérationnelle s'en trouvent par la suite facilitées. Il ne s'agit pas seulement de processus ponctuels; en règle générale, une information bien communiquée se réfère aussi aux valeurs à partager, durablement, que ce soit dans une entreprise, une association ou un service public.

Un autre chef d'entreprise (alsacienne, de 150 salariés) a souligné les bienfaits de la communication en termes de créativité de nouveaux biens et services. En effet, depuis une quinzaine d'années on assiste, grâce à Internet et aux technologies numériques, à une révolution « extraordinaire " dans le domaine de la communication qui se traduit par un «foisonnement », jusqu'ici inconnu, de données, d'informations, d'idées, de relations. Chacun peut en tirer profit. Ce foisonnement a amené de la vitesse, de l'imagination, de l'efficacité. Le bilan est indiscutablement positif. Cette "révolution » a réduit l'importance de la communication verticale, au profit d'une organisation par projet et d'une communication horizontale. Comment faire pour que "ça marche »? Dans l'industrie, comme ailleurs, il faut changer les méthodes de pensée, faire confiance aux hommes, et fixer des règles procédurales claires, précisant ce qu'il est permis de faire, de dire, de ne pas faire, de ne pas dire, dans telle ou telle circonstance.

Le contenu de la communication a été évoqué en filigrane dans les témoignages ci-dessus. Plus généralement il faut retenir quelques points essentiels:

La communication ne saurait être figée; elle doit incessamment être construite et reconstruite en fonction des besoins qui évoluent. Tantôt il s'agit de donner la priorité à l'information, tantôt la communication est centrée sur la volonté de nouer des liens entre les personnes, tantôt elle cible surtout l'efficacité opérationnelle, tantôt elle est plutôt orientée vers l'animation d'équipes ou l'explicitation d'un changement organisationnel. Les deux vecteurs de la construction sont d'une part, la gouvernance (qui définit où, comment et par qui les décisions se prennent) et d'autre part, l'« organisation » (chargée de la mise en œuvre des responsabilités et des process pour arriver 
au résultat désiré). Mais la communication ne trouve sa véritable efficacité que dans le lien individuel et la coopération entre équipes. L’efficacité suppose en la matière de l'empathie (la faculté de s'identifier à quelqu'un d'autre), de la compétence émotionnelle et de la compétence sociale de la part des acteurs en jeu. L'efficacité suppose aussi l'interactivité aux différents niveaux de la hiérarchie.

Par ailleurs, la communication doit veiller à la cohérence des nombreux signaux émis, selon des formes horizontales, verticales, diagonales, internes, externes (toutes interactives) présentées ci-dessus. Sinon la dynamique de la communication va se trouver affaiblie. L'exercice est particulièrement complexe et délicat dans les grandes structures institutionnelles (entreprises multinationales, grandes organisations publiques); il est facilité dans les petites structures (telles les PME) où le chef d'entreprise, décideur, se trouve en situation de proximité avec sa force de travail opérationnelle. Dans tous les cas, "le manager doit avoir du souffle pour stimuler la réussite »!

La communication ne saurait ignorer les exigences éthiques. On peut à cet égard, au confluent de la morale et de la raison, faire plusieurs observations. La première concerne l'exigence de vérité, die Wahrheit. Cette exigence est liée au respect que l'on doit à toute personne; elle est aussi le support de la confiance entre les individus, de la reconnaissance recherchée par tout un chacun. Le mensonge est suicidaire. La fiabilité de la communication est liée à la clarté et à la crédibilité du message, à sa "transparence ", sa traçabilité. Le respect des règles éthiques permet aussi d'engager utilement des discussions sur les mésaventures, les ratages ou les éléments perturbateurs (Störfälle) de la communication, et les mesures à prendre à leur encontre ou à leur suite. Les échecs, les erreurs ne sont certes pas souhaitables mais riches d'enseignements à prendre en considération. La seconde observation concerne le respect dû aux personnes, collaborateurs ou partenaires. L'expansion exponentielle des moyens de communication, combinée à la facilité de leur utilisation et à leur faible coût, est en voie de ruiner la vie privée des personnes. Ordinateurs, tablettes, téléphones portables, " smartphones ", etc. sont à même d'orchestrer un véritable harcèlement de communications, jour et nuit puisque grâce à la globalisation il y a toujours des lieux d'activité dans le monde susceptibles de réclamer des liens de communication à tout moment. Le temps pour la vie privée, éloignée du travail, se réduit comme une peau de chagrin et vient perturber les équilibres physique, psychique et nerveux des personnes. Les dommages qui en résultent ne sont pas seulement individuels mais aussi sociaux. L'abondance de biens nuit. La pensée souffre des excès de vitesse et de l'agitation perpétuelle que créent tous les messages, forcément décousus, qui sont émis par les médias de toute sorte et qui, comme les lois, demandent à ne pas être ignorés. L'abondance de tous ces signaux de communication, privés ou publics, a au moins deux effets regrettables: une incitation à la superficialité (Oberflächlichkeit) et une grande fatigue! Un vaste champ de recherche de solutions est ouvert. Quels excès peut-on en réalité limiter dans un monde libertaire et techniquement difficile à contrôler d'une manière acceptable?

La conclusion que l'on est à même de retenir des échanges d'idées, de convictions, et d'expériences intervenus durant cette journée de séminaire est relativement optimiste. Elle pourrait se résumer comme suit:

L'être humain est une valeur en soi, la plus précieuse; il est capable de créer de la valeur socio-économique; il est capable de penser les valeurs du futur. 
On retrouve par là le bien-fondé et l'actualité d'une Pensée de Blaise Pascal, écrite au $\mathrm{XVII}^{\mathrm{e}}$ siècle: "L'homme n'est qu'un roseau [...] mais c'est un roseau pensant. Il ne faut pas que l'univers entier s'arme pour l'écraser. [...] Toute notre dignité consiste en la pensée. Travaillons donc à bien penser : voilà le fondement de la morale. »Cette Pensée doit cependant être complétée par une des affirmations fortes de la journée (centrée sur les témoignages de terrain); elle devient alors « Travaillons à bien penser et à bien agir: voilà le fondement de la morale».

Pour y parvenir à ce «bien penser » et à ce «bien agir » il importe de se rappeler des vertus du dialogue, à tous les niveaux. Cela suppose que soient dégagés dans la vie active des espaces et des temps de discussion. Mais un dialogue n'est vraiment fécond que dans la mesure où les participants concernés font preuve d'ouverture d'esprit, d'empathie et acceptent de prendre honnêtement en considération les vraies questions qui se posent sur le terrain. Le dialogue conduira alors au partage de valeurs communes et à la reconnaissance individuelle; il conduira aussi à la construction et à la réussite de projets engagés ensemble.

Le caractère infiniment précieux de l'homme impose à chaque acteur de la vie sociale de respecter l'être humain, sa dignité et sa noblesse, de ne pas gaspiller inutilement son propre temps et son énergie, pas plus que celui de l'autre. En d'autres termes, la vie sociale impose un principe de responsabilité réciproque. C'est une attitude nécessaire pour créer un climat de confiance, que ce soit dans l'entreprise ou dans d'autres sphères de la vie civique. C'est la confiance générée qui permet de gérer la complexité de la vie sociale, avec ses forces inégales voire contradictoires, et d'éviter ainsi des conflits sociaux dommageables. Mais en tout état de cause la confiance doit se mériter et se justifier. L'éducation, la formation, la culture sociale ont à cet égard un rôle majeur; les témoignages de réalités différentes de part et d'autre du Rhin ont été éclairants à ce sujet.

Dans un cadre de vie démocratique, où chacun se voit reconnaître une certaine liberté de manœuvre, il reste un problème collectif important: celui de la coordination des initiatives individuelles dans le respect des intérêts de tous, en d'autres termes, d'une nécessaire solidarité entre les membres d'un groupe. C'est là le fondement du bien-être individuel, pouvant alors s'épanouir dans un contexte collectif pacifique. C'est aussi une condition indispensable à l'émergence d'un « développement durable » respectueux du bien-être des générations futures, des ressources naturelles (un bien commun de l'humanité) et d'une saine vitalité de l'environnement planétaire. Ce n'est que dans un contexte de développement véritablement «durable » que l'humain pourra être réellement préservé et promu dans la vie socio-économique. 